\section{RICYDE. Revista Internacional de Ciencias del Deporte doi: $10.5232 /$ ricyde \\ Rev. Int. cienc. deporte}

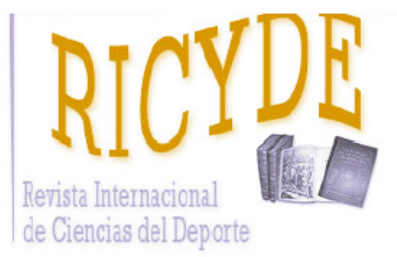

RICYDE. Revista Internacional de Ciencias del Deporte Volumen XV - Año XV

Paginas: $307-322$ - ISSN: $1885-3137$

Número 58 - Octubre 2019

https://doi.org/10.5232/ricyde2019.05801

\title{
Asociación entre fuerza de prensión manual y bienestar en mujeres con fibromialgia Association of handgrip strength and well-being in women with fibromyalgia
}

\section{Cristina Maestre-Cascales ${ }^{1}$, María José Girela-Rejónn2, Diego Pedro Sánchez-Gallo ${ }^{3}$, Pedro Acosta Manzano ${ }^{4}$,} Blanca Gavilán-Carrera ${ }^{4}$, Inma García-Rodríguez ${ }^{4}$ Jesús Javier Rojo-González ${ }^{1}$, Virginia Ariadna Aparicio ${ }^{5}$

1.Departamento de Salud y Rendimiento Humano, Facultad de Ciencias del Deporte, Universidad Politécnica de Madrid, España.

2.Departamento de Didáctica de la Expresión Corporal, Facultad de Ciencias de la Educación, Universidad de Granada, España.

3.Departamento de Fisiología, Facultad de Farmacia, Facultad de Ciencias del Deporte, e Instituto de Nutrición y Tecnología de los Alimentos, Granada. España 4.Departamento de Educación Física y Deportiva, Facultad de Ciencias del Deporte, Universidad de Granada, España.

5 Departamento de Fisiología, Centro de Investigación Biomédica e Instituto Mixto Universitario Deporte y Salud. Universidad de Granada. España

\section{Resumen}

La fibromialgia es una enfermedad caracterizada por una sintomatología compleja con presencia de dolor crónico generalizado junto con otros síntomas incapacitantes como fatiga y problemas de memoria. Como consecuencia, suelen verse deteriorados sus niveles de bienestar. Por lo tanto, es necesario encontrar factores modificables mediante terapia para mejorar el bienestar en la fibromialgia. En esta población, la fuerza de prensión manual es un conocido marcador relevante de salud física, pero se desconoce si también lo es de salud psicológica. Objetivo. Analizar la asociación entre los niveles de fuerza de prensión manual con el bienestar subjetivo en mujeres con fibromialgia. Métodos. Este estudio transversal incluyó a 465 mujeres pertenecientes a pertenecientes a la Comunidad de Andalucía (España). La fuerza se midió a través del test de prensión manual. Las dos dimensiones (i.e., afectiva y cognitiva) del bienestar subjetivo se midieron con cuestionarios, Positive and Negative Affect Scale (PANAS) para el afecto positivo y negativo y Satisfaction with Life Scale (SWLS) para la satisfacción con la vida. La asociación de la fuerza de prensión manual con estas dimensiones se analizó mediante regresiones lineales ajustadas por la edad y el consumo de medicamentos. Resultados. Mayores niveles de fuerza se asociaron con mayor afecto positivo $(p<0,001)$, menor afecto negativo $(p<0,001)$ y mayor satisfacción con la vida $(p<0,05)$. Conclusión. En una muestra representativa de la población andaluza de mujeres con fibromialgia, este estudio ha identificado que los niveles de fuerza están positivamente asociados con puntuaciones más favorables de bienestar subjetivo (i.e., afecto positivo, afecto negativo y satisfacción con la vida). En general, la fuerza de estas asociaciones fue débil. Para esclarecer la causalidad de nuestros hallazgos se requieren futuros estudios longitudinales y experimentales.

Key words: actividad física, condición física, estado de ánimo, felicidad, fibromialgia.

\section{Abstract}

Fibromyalgia is a disease characterized by a complex symptomatology including presence of widespread chronic pain and other disabling symptoms such as fatigue and poor memory. Thus, fibromyalgia often supposes a considerable burden for well-being. Therefore, finding factors that may be modifiable by therapy is of interest in order to enhance well-being among people with fibromyalgia. Objective. To analyse the association between levels of handgrip strength with well-being in women with fibromyalgia. Methods. This cross-sectional study included 465 women with fibromyalgia from from Community of Andalusia (Spain). Strength was measured by means of the handgrip strength test. The two dimensions (affective and cognitive) of subjective well-being were measured with questionnaires, positive and negative affect was assessed by the Positive and Negative Affect Scale (PANAS) and satisfaction with life by the Satisfaction with Life Scale (SWLS). The association between the handgrip strength test and positive affect, negative affect and satisfaction with life was analysed by linear regression models adjusted for age and consumption of medications (for depression, pain and sleep). Results. Higher levels of strength were associated with higher positive affect $(p<0.001)$, lower negative affect $(p<0.001)$ and higher life satisfaction $(p<0.05)$. Conclusion. Conducted in a representative sample of the Andalusian population of women with fibromyalgia, the present study has identified that muscular strength levels are positively associated with more favorable scores in all dimensions of subjective well-being (i.e., positive affect, negative affect, and life satisfaction). Overall, the strength of these associations was weak. In order to elucidate the causality of our findings, future longitudinal and experimental studies are required.

Palabras clave: fibromyalgia, happiness, physical activity, physical fitness, physical function.

Correspondencia/correspondence: María José Girela-Rejón.

Departamento de Didáctica de la Expresión Corporal, Facultad de Ciencias de la Educación, Universidad de Granada, España. Email: mjgirela@ugr.es 
Maestre-Cascales, C.; Girela-Rejón, M. J.; Sánchez-Gallo, D. P.; Acosta-Manzano, D.; Gavilán-Carrera, B.; García-Rodríguez, I.; Rojo-González, J. J., y Aparicio, V. A. (2019). Asociación entre fuerza de prensión manual y bienestar en mujeres con fibromialgia. RICYDE. Revista internacional de ciencias del deporte. 58(15), 307-322. https://doi.org/10.5232/ricyde2019.05801

\section{Introducción}

$\mathrm{L}$ a fibromialgia es una enfermedad que se caracteriza por la presencia de dolor crónico, acompañada por otros síntomas como niveles elevados de fatiga, dificultades cognitivas y sueño no reparador (Clauw, 2014). Por ello, la fibromialgia supone una carga elevada para los pacientes, siendo un problema de salud pública de primer orden ya que genera altas demandas en el sistema sanitario y supone un incremento relevante en los costes económicos (SicrasMainar, Rejas, Navarro, Blanca, Morcillo, Larios, y Villarroya, 2009). Investigaciones recientes se han centrado en aspectos emocionales de la fibromialgia. En concreto, se ha mostrado que las personas con fibromialgia tienen niveles muy deteriorados de bienestar subjetivo (Hassett, Simonelli, Radvanski, Buyske, Savage, y Sigal, 2008; van Middendorp, Lumley, Jacobs, Bijlsma y Geenen, 2010). Además, los niveles bajos de bienestar están claramente asociados a la gravedad de la fibromialgia, la fatiga y el dolor (Estevez-Lopez, Segura-Jimenez, Alvarez-Gallardo, Borges-Cosic, Pulido-Martos, Carbonell-Baeza, y Delgado-Fernandez, 2017; van Middendorp y col., 2010).

Actualmente ha aumentado el interés por mejorar la aceptación personal y desarrollar estrategias de adaptación a la enfermedad. Esto requiere un proceso dinámico necesario para reequilibrar las nuevas circunstancias y el correcto mantenimiento de las funciones psicológicas (Stanton, Revenson y Tennen, 2007). Tradicionalmente, la investigación se ha centrado en respuestas no adaptativas (factores de vulnerabilidad) como, por ejemplo, la catastrofización del dolor que se define como la tendencia de prestar excesiva atención a las sensaciones de dolor (Estevez-Lopez, 2018; López, 2018). Sin embargo, los factores adaptativos, deseables para gestionar bien la fibromialgia, han recibido menos atención. Entre estos factores, conocidos como resiliencia, el bienestar subjetivo es uno de los más importantes. El bienestar subjetivo se define como la evaluación de la vida tanto a nivel afectivo (e.g., afecto positivo y afecto negativo) como cognitivo (e.g., satisfacción con la vida) (Diener, Suh, Lucas, y Smith, 1999). Entre estas dimensiones del bienestar subjetivo, el afecto positivo es de especial relevancia para favorecer la adaptación a esta patología crónica (Estévez-López, Gray, SeguraJiménez, Soriano-Maldonado, Álvarez-Gallardo, Arrayás-Grajera, y Pulido-Martos, 2015).

Dado que el bienestar subjetivo en personas con fibromialgia es, en promedio, bajo (Hassett y col., 2008), es necesario encontrar factores modificables que estén relacionados con el bienestar subjetivo para adaptar y focalizar mejor los programas de intervención en fibromialgia. En este contexto, Mannerkorpi, Svantesson y Broberg (2006) sugirieron que los test de condición física podrían servir como complemento a las herramientas actuales empleadas en la valoración clínica cuando se planifica el tratamiento orientado a pacientes con fibromialgia. Estos mismos autores, indicaron que la fuerza de prensión manual es de especial interés en fibromialgia. Desde un punto de vista clínico, nuestro grupo ha mostrado la capacidad diagnóstica y pronostica del test de fuerza de prensión manual para valorar la presencia y severidad de esta patología (Aparicio, Carbonell-Baeza, Ruiz, Aranda, Tercedor, Delgado-Fernandez, y Ortega, 2013; Aparicio, Ortega, Heredia, Carbonell-Baeza, Sjöström, y Delgado-Fernandez, 2011; Aparicio, Segura-Jimenez, Alvarez-Gallardo, Soriano-Maldonado, Castro-Pinero, DelgadoFernandez, y Carbonell-Baeza, 2015; Castro-Piñero, Aparicio, Estévez-López, ÁlvarezGallardo, Borges-Cosic, Soriano-Maldonado, y Segura-Jiménez, 2017). Actualmente, se considera el test de fuerza de prensión manual como un importante marcador de salud en fibromialgia (Aparicio y col., 2013; Aparicio y col., 2011; Aparicio y col., 2015). Sin embargo, este test de fuerza de prensión manual no ha sido estudiado en relación con el bienestar subjetivo de las personas con fibromialgia. 
Maestre-Cascales, C.; Girela-Rejón, M. J.; Sánchez-Gallo, D. P.; Acosta-Manzano, D.; Gavilán-Carrera, B.; García-Rodríguez, I.; Rojo-González, J. J., y Aparicio, V. A. (2019). Asociación entre fuerza de prensión manual y bienestar en mujeres con fibromialgia. RICYDE. Revista internacional de ciencias del deporte. 58(15), 307-322. https://doi.org/10.5232/ricyde2019.05801

Por lo tanto, el test de fuerza de prensión manual es un marcador de salud física, pero se desconoce si también lo es de salud psicológica, en general, y del bienestar subjetivo, en particular. Por ello, el objetivo del presente estudio ha sido evaluar la asociación de los niveles de fuerza con el bienestar subjetivo en mujeres con fibromialgia.

\section{Material y método}

\section{Participantes}

La metodología y el procedimiento de muestreo que se utilizó en este estudio transversal aparece detallado previamente en el proyecto X (Segura-Jimenez, Alvarez-Gallardo, CarbonellBaeza, Aparicio, Ortega, Casimiro, y Delgado-Fernandez, 2015a). El proceso de reclutamiento de los participantes se llevó a cabo mediante dos vías; la primera, a través de la Federación Andaluza de Fibromialgia, contactando con las correspondientes asociaciones locales de las ocho provincias que abarca la comunidad autónoma de Andalucía y la segunda, vía telefónica, postal, por correo electrónico, prensa digital y a través de universidades.

Un total de 646 pacientes adultos con fibromialgia se ofrecieron como voluntarios para participar en el estudio. Este número de participantes superó el mínimo establecido para obtener una muestra representativa de la población Andaluza con fibromialgia (Segura-Jimenez y col., 2015a). Dada la transcendencia social que este estudio tuvo, el equipo investigador decidió no restringir la participación de ninguna persona que cumpliese los criterios de inclusión. Todos los participantes fueron informados sobre los objetivos y cumplieron con los siguientes criterios de inclusión; a) haber sido previamente diagnosticados de fibromialgia por un reumatólogo, y b) cumplir los criterios de diagnóstico para la fibromialgia según el Colegio Americano de Reumatología (ACR) (Wolfe, Smythe, Yunus, Bennett, Bombardier, Goldenberg, Clark, 1990), lo que fue corroborado por los investigadores del proyecto al-Ándalus. Antes de participar en el estudio, todos ellos firmaron el consentimiento informado por escrito.

El protocolo del proyecto X fue aprobado por el Comité de Ética del X; Número de registro: X. Además, se siguieron las pautas éticas de la Declaración de Helsinki (modificada en 2008).

\section{Instrumentos}

\section{Datos sociodemográficos}

La información sociodemográfica de los participantes fue registrada mediante una entrevista inicial donde se recogieron datos como la fecha de nacimiento, el estado civil y nivel educativo. Para finalizar, debían responder a la siguiente cuestión: “¿Alguna vez le han diagnosticado una enfermedad aguda o terminal?” siendo este un criterio de exclusión.

\section{Consumo de fármacos}

Los participantes indicaron de forma dicotómica (si/no) si ingerían fármacos para el dolor, depresión y sueño/relajación.

\section{Índice de Masa Corporal (IMC)}

Se midió el peso en kilogramos (InBody R20, Biospace, Seoul, Sur Korea) y altura en centímetros (Seca 22). Para calcular el Índice Masa Corporal (IMC) se utilizó la fórmula matemática $\mathrm{kg} / \mathrm{m}^{2}$ 
Maestre-Cascales, C.; Girela-Rejón, M. J.; Sánchez-Gallo, D. P.; Acosta-Manzano, D.; Gavilán-Carrera, B.; García-Rodríguez, I.; Rojo-González, J. J., y Aparicio, V. A. (2019). Asociación entre fuerza de prensión manual y bienestar en mujeres con fibromialgia. RICYDE. Revista internacional de ciencias del deporte. 58(15), 307-322. https://doi.org/10.5232/ricyde2019.05801

\section{$\underline{\text { Rendimiento cognitivo }}$}

El deterioro cognitivo se midió a través de la escala Mini Mental Examination (MMSE) (Lobo, Ezquerra, Gómez, Sala y Seva, 1979), compuesta por siete categorías: orientación espacial, orientación temporal y concentración, recuerdo, lenguaje y construcción visual. La puntuación de MMSE oscila entre 0 a 30, por lo tanto, las puntuaciones más bajas reflejan un mayor deterioro cognitivo. Los participantes con deterioro cognitivo severo (puntuación $\leq 10$ ) fueron excluidos.

\section{Puntos de dolor}

Los puntos de dolor fueron evaluados siguiendo los criterios del Colegio Americano de Reumatología en 1990 (American College of Rheumatology, ACR-1990; Segura-Jiménez y col., 2014; Wolfe y col., 1990) para el diagnóstico y clasificación de la fibromialgia. Por otro lado, se utilizó un algómetro de presión estándar (FPK 20; Wagner Instruments, Greenwich, CT, USA). Se registró el total de puntos sensibles al dolor para cada participante.

\section{Bienestar subjetivo (PANAS) y satisfacción con la vida (SWLS)}

El bienestar subjetivo se midió a través del cuestionario Positive and Negative Affect Scale (PANAS) (Watson, Clark y Tellegen, 1988), diseñado para evaluar el afecto positivo y afecto negativo. Este cuestionario abarca 20 ítems, 10 relacionados con afecto positivo (entusiasmado, etc.) y los otros 10 con afecto negativo (asustado, etc.). Los participantes respondieron cada ítem mediante una escala tipo Likert: 1) muy ligeramente o nada, 2) un poco, 3) moderadamente, 4) bastante, y 5) extremadamente. El marco temporal adoptado fue en "general". Los ítems del PANAS son: Interesado, angustiado, excitado, molesto, fuerte, culpable, asustado, hostil, entusiasta, orgulloso, irritable, alerta, avergonzado, inspirado, nervioso, determinado, atento, nervioso, activo y asustado. El rango de puntuación es de 10-50 tanto para el afecto positivo como para el afecto negativo. Para la satisfacción con la vida se utilizó la escala SWLS (Satisfaction with Life Scale), la cual abarca una puntuación que varía entre 5-25.

\section{Test de fuerza de prensión manual}

El test de prensión manual es válido, fiable y viable (Nordenskiold y Grimby, 1993; Valkeinen, Alen, Hakkinen, Hannonen, Kukkonen-Harjula, y Hakkinen, 2008) ya que proporciona información útil sobre la fuerza muscular general del participante. Se evaluó mediante el dinamómetro de mano TKK ya que el sesgo sistémico es pequeño y ha demostrado tener los resultados más fiables cuando se utilizan mediciones repetidas con pesos conocidos. El rango de agarre es ajustable, para ello se utilizó la fórmula sugerida por Ruiz, España-Romero, Ortega, Sjöström, Castillo, y Gutierrez (2006) para calcular la distancia óptima de agarre. El sujeto ejerció presión de forma gradual manteniendo al menos 2 segundos, realizando el test con la mano derecha y a continuación con la izquierda. Cada paciente realizó dos intentos con cada mano, colocando el brazo completamente extendido y formando un ángulo de $30^{\circ}$ con respecto al tronco. Se registró la puntuación máxima en kilogramos $(\mathrm{kg})$ de cada mano y se utilizó la puntuación media de la mano izquierda y derecha para los análisis

\section{Procedimiento}

Las pruebas, test y cuestionarios se realizaron en un orden determinado, el cual garantiza que los resultados no se viesen alterados. Estas, se llevaron a cabo en tres días consecutivos, siendo el primer día destinado a la evaluación del rendimiento cognitivo (MMSE), datos sociodemográficos, composición corporal (IMC; $\mathrm{Kg} / \mathrm{m}^{2}$ ) y la corroboración del diagnóstico siguiendo los criterios del ACR-1990. En el segundo día, los participantes cumplimentaron de 
Maestre-Cascales, C.; Girela-Rejón, M. J.; Sánchez-Gallo, D. P.; Acosta-Manzano, D.; Gavilán-Carrera, B.; García-Rodríguez, I.; Rojo-González, J. J., y Aparicio, V. A. (2019). Asociación entre fuerza de prensión manual y bienestar en mujeres con fibromialgia. RICYDE. Revista internacional de ciencias del deporte. 58(15), 307-322. https://doi.org/10.5232/ricyde2019.05801

forma autónoma los cuestionarios de PANAS y SWLS. Para finalizar, en el tercer día realizaron las mediciones de fuerza manual.

\section{Análisis estadístico}

La frecuencia y el porcentaje o la media y la desviación típica se utilizaron para informar de la estadística descriptiva de las variables categóricas y continuas, respectivamente. Por otro lado, la asociación entre el test de fuerza de prensión manual y las puntuaciones de bienestar subjetivo se analizaron mediante el análisis de regresión lineal. Antes de proceder con este análisis, se comprobó que los supuestos de linealidad, normalidad y homocedasticidad (e independencia de errores) se cumplieron. En los modelos de regresión lineal, la fuerza de presión manual fue la variable independiente. La edad y la medicación para el dolor, depresión, relajación o sueño se introdujeron como variables confusoras (paso 1). Se exploró el posible papel confusor del IMC y el peso, pero ninguno jugo dicho papel por lo que no fueron incluidos en el modelo. Posteriormente en el paso 2, afecto positivo, afecto negativo y satisfacción con la vida entraron como variable dependiente en tres modelos de regresión lineal (uno por variable dependiente). Dichos análisis se llevaron a cabo utilizando el paquete estadístico para Ciencias Sociales (IBM SPSS for Mac, versión 20.0; Armonk, NY, USA). En todos los análisis el nivel de significación estadística fijado fue de $\mathrm{p}<0,05$.

\section{Resultados}

De 646 posibles participantes, 181 fueron excluidos por diferentes motivos: 39 no estaban diagnosticados por un reumatólogo, 99 no cumplieron los criterios de ACR-1990, 21 eran hombres, 1 mostró un rendimiento cognitivo deteriorado, 2 presentaron alguna enfermedad aguda o severa, 1 no tenía datos de composición corporal y 18 no realizaron el test de prensión manual. La figura 1 muestra el diagrama de flujo del estudio. 
Maestre-Cascales, C.; Girela-Rejón, M. J.; Sánchez-Gallo, D. P.; Acosta-Manzano, D.; Gavilán-Carrera, B.; García-Rodríguez, I.; Rojo-González, J. J., y Aparicio, V. A. (2019). Asociación entre fuerza de prensión manual y bienestar en mujeres con fibromialgia. RICYDE. Revista internacional de ciencias del deporte. 58(15), $307-322$. https://doi.org/10.5232/ricyde2019.05801

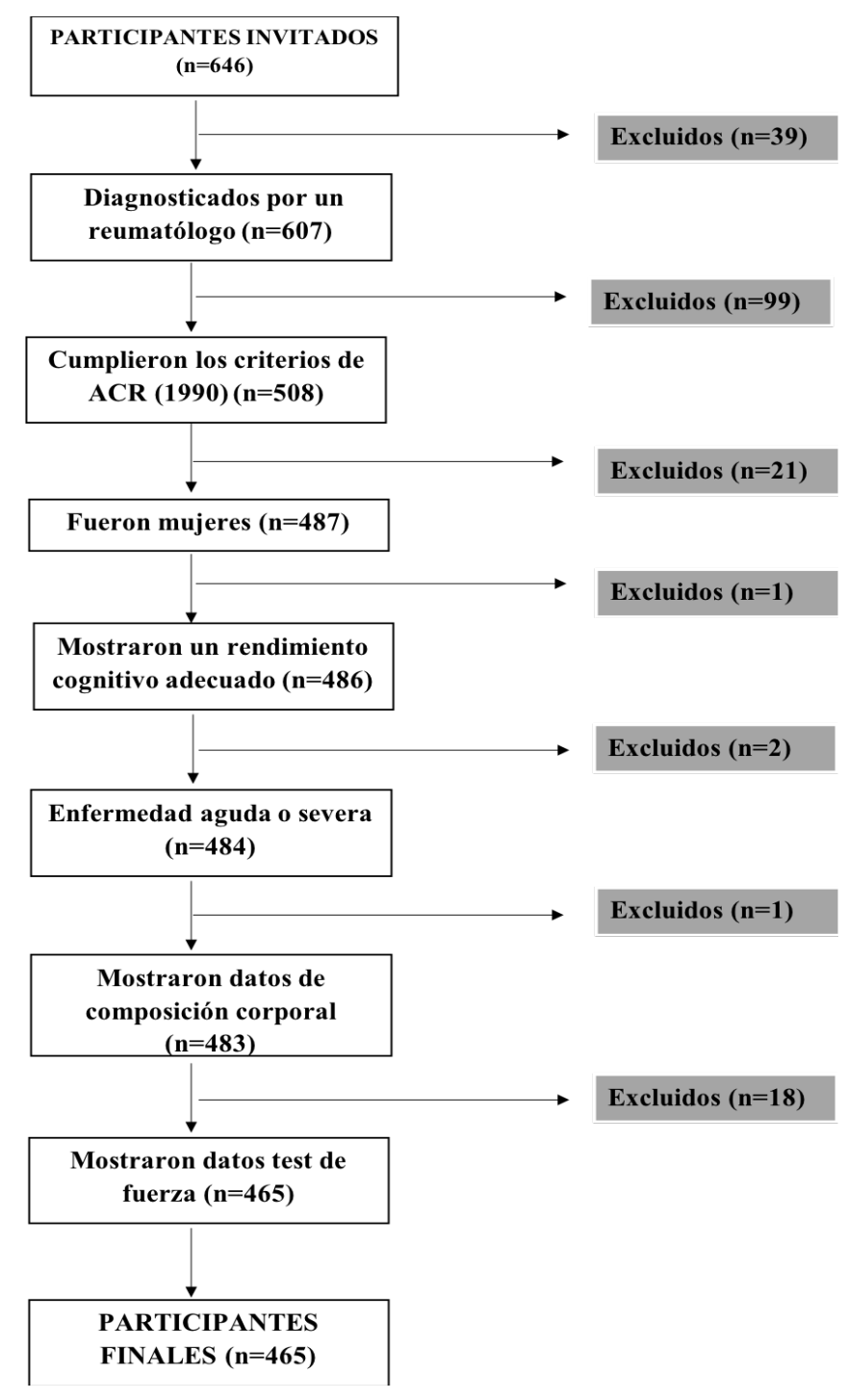

Figura 1. Diagrama de flujo del estudio

Las características descriptivas de los 465 que formaron parte finalmente del estudio se presentan en la tabla 1. Las Tablas 2, 3 y 4 muestran los resultados procedentes del análisis de regresión lineal entre los niveles de fuerza y el afecto positivo, el afecto negativo y la satisfacción con la vida. Entre ellos encontramos que un mayor nivel de fuerza manual está asociado con mayor afecto positivo $(t=3,26 ; p<0,01)$, menor afecto negativo $(t=-3,95 ; p<0,001)$ y mayor satisfacción con la vida $(\mathrm{t}=2,57 ; \mathrm{p}<0,05)$. El modelo final explicó entre el $6 \%$ y $11 \%$ de variabilidad en las dimensiones del bienestar subjetivo [los valores de $\mathrm{R}^{2}$ ajustados fueron $0,09, p<0,01$, para el afecto positivo; $0,11, p=0,001$ para el afecto negativo y $0,06, p<0,05$ para la satisfacción con la vida]. 
Maestre-Cascales, C.; Girela-Rejón, M. J.; Sánchez-Gallo, D. P.; Acosta-Manzano, D.; Gavilán-Carrera, B.; García-Rodríguez, I.; Rojo-González, J. J., y Aparicio, V. A. (2019). Asociación entre fuerza de prensión manual y bienestar en mujeres con fibromialgia. RICYDE. Revista internacional de ciencias del deporte. 58(15), $307-322$. https://doi.org/10.5232/ricyde2019.05801

Tabla 1. Características de las participantes en el estudio $(n=465)$

\begin{tabular}{|c|c|c|}
\hline Variables & Media & DT \\
\hline Edad & 52,1 & 7,9 \\
\hline Altura (cm) & 157,83 & 6,03 \\
\hline Peso $(\mathrm{kg})$ & 71,16 & 13,78 \\
\hline Índice Masa Corporal (kg/m²) & 28,5 & 5,3 \\
\hline Puntos de dolor: número total & 16,7 & 1,9 \\
\hline \multicolumn{3}{|l|}{ Condición física } \\
\hline Test de prensión manual (kg) & 19,06 & 6,5 \\
\hline \multicolumn{3}{|l|}{ Componentes del bienestar subjetivo } \\
\hline Afecto positivo (PANAS; 10-50) & 22,9 & 6,7 \\
\hline Afecto Negativo (PANAS; 10-50) & 24,0 & 8,4 \\
\hline \multirow[t]{2}{*}{ Satisfacción con la vida (SWLS; 5-25) } & 14,0 & 4,6 \\
\hline & Frecuencia & $\%$ \\
\hline \multicolumn{3}{|l|}{ Estado civil } \\
\hline Casada & 312 & 75,7 \\
\hline Soltera & 36 & 7,7 \\
\hline Separada/divorciada & 52 & 11,2 \\
\hline Viuda & 25 & 5,4 \\
\hline \multicolumn{3}{|l|}{ Nivel de educación } \\
\hline Estudios sin finalizar & 50 & 10,8 \\
\hline Primaria & 225 & 40,8 \\
\hline Secundaria y FP & 126 & 27,1 \\
\hline Título universitario & 64 & 13,8 \\
\hline
\end{tabular}

En el paso 1, se incluyeron las variables confusoras y en el paso 2, la variable independiente de interés.

B y $\beta$, Coeficientes de regresión no estandarizados y estandarizados con los niveles de significación basados en el estadístico t, respectivamente; IC, Intervalo de Confianza; Adj.R2, R2 ajustado con los niveles de significación basados en el cambio de $\mathrm{F}$.

$* \mathrm{p}<0,05 ; * * \mathrm{p}<0,01 ; * * * \mathrm{p}<0,001$ 
Maestre-Cascales, C.; Girela-Rejón, M. J.; Sánchez-Gallo, D. P.; Acosta-Manzano, D.; Gavilán-Carrera, B.; García-Rodríguez, I.; Rojo-González, J. J., y Aparicio, V. A. (2019). Asociación entre fuerza de prensión manual y bienestar en mujeres con fibromialgia. RICYDE. Revista internacional de ciencias del deporte. 58(15), 307-322. https://doi.org/10.5232/ricyde2019.05801

Tabla 2. Asociación entre fuerza de prensión manual y el afecto positivo $(\mathrm{n}=465)$

\begin{tabular}{|c|c|c|c|c|c|}
\hline Pasos & B & 95\% IC: Límite Inferior & $\begin{array}{l}\text { 95\% IC: Límite } \\
\text { Superior }\end{array}$ & $\boldsymbol{\beta}$ & $\operatorname{Adj} R^{2}$ \\
\hline 1 & & & & & $0,07 * * *$ \\
\hline Edad (años) & & 0,23 & 0,17 & $0,11 *$ & \\
\hline Medicamentos para la depresión & & $-3,32$ & $-0,70$ & $-0,14 * *$ & \\
\hline Medicamentos para el dolor & & $-4,52$ & $-0,45$ & $-0,10^{*}$ & \\
\hline Medicamentos para la relajación o el sueño & & $-3,04$ & $-0,12$ & $0,15^{*}$ & \\
\hline \multicolumn{6}{|l|}{2} \\
\hline Test de prensión manual (kg) & $0,09 * *$ & 0,06 & 0,25 & $0,15 * *$ & $0,09 * *$ \\
\hline
\end{tabular}

En el paso 1, se incluyeron las variables confusoras y en el paso 2, la variable independiente de interés.

B y $\beta$, Coeficientes de regresión no estandarizados y estandarizados con los niveles de significación basados en el estadístico t, respectivamente; IC, Intervalo de Confianza; Adj.R2, R2 ajustado con los niveles de significación basados en el cambio de F.

$* \mathrm{p}<0,05 ; * * \mathrm{p}<0,01 ; * * * \mathrm{p}<0,001$ 
Maestre-Cascales, C.; Girela-Rejón, M. J.; Sánchez-Gallo, D. P.; Acosta-Manzano, D.; Gavilán-Carrera, B.; García-Rodríguez, I.; Rojo-González, J. J., y Aparicio, V. A. (2019). Asociación entre fuerza de prensión manual y bienestar en mujeres con fibromialgia. RICYDE. Revista internacional de ciencias del deporte. 58(15), 307-322. https://doi.org/10.5232/ricyde2019.05801

Tabla 3. Asociación entre fuerza de prensión manual y el afecto negativo $(\mathrm{n}=465)$

\begin{tabular}{|c|c|c|c|c|c|}
\hline Pasos & B & $\begin{array}{l}\text { 95\% IC: Límite } \\
\text { Inferior }\end{array}$ & 95\% IC: Límite Superior & $\boldsymbol{\beta}$ & $\operatorname{Adj} R^{2}$ \\
\hline 1 & & & & & $0,08 * * *$ \\
\hline Edad (años) & $-0,22$ & $-0,32$ & $-0,13$ & $-0,21 * * *$ & \\
\hline Medicamentos para la depresión & 1,78 & 0,16 & 3,40 & $0,10^{*}$ & \\
\hline Medicamentos para el dolor & 0,68 & $-1,82$ & 3,19 & 0,02 & \\
\hline Medicamentos para la relajación o el sueño & 2,84 & 1,04 & 4,65 & $0,15 * *$ & \\
\hline \multicolumn{6}{|l|}{2} \\
\hline Test de prensión manual (kg) & $-0,23$ & $-0,35$ & $-0,11$ & $-0,18 * * *$ & $0,11 * * *$ \\
\hline
\end{tabular}

En el paso 1, se incluyeron las variables confusoras y en el paso 2, la variable independiente de interés.

B y $\beta$, Coeficientes de regresión no estandarizados y estandarizados con los niveles de significación basados en el estadístico t, respectivamente; IC, Intervalo de Confianza;

Adj.R2, R2 ajustado con los niveles de significación basados en el cambio de $\mathrm{F}$.

$* \mathrm{p}<0,05 ; * \mathrm{p}<0,01 ; * * * \mathrm{p}<0,001$ 
Maestre-Cascales, C.; Girela-Rejón, M. J.; Sánchez-Gallo, D. P.; Acosta-Manzano, D.; Gavilán-Carrera, B.; García-Rodríguez, I.; Rojo-González, J. J., y Aparicio, V. A. (2019). Asociación entre fuerza de prensión manual y bienestar en mujeres con fibromialgia. RICYDE. Revista internacional de ciencias del deporte. 58(15), 307-322. https://doi.org/10.5232/ricyde2019.05801

Tabla 4. Asociación entre fuerza de prensión manual y la satisfacción con la vida $(\mathrm{n}=465)$

\begin{tabular}{|c|c|c|c|c|c|}
\hline Pasos & B & $\begin{array}{l}\text { 95\% IC: Límite } \\
\text { Inferior }\end{array}$ & $\begin{array}{l}\text { 95\% IC: Límite } \\
\text { Superior }\end{array}$ & $\boldsymbol{\beta}$ & $\operatorname{Adj} R^{2}$ \\
\hline 1 & & & & & $0,05 * * *$ \\
\hline Edad (años) & 0,58 & $6 \cdot 10^{-2}$ & 0,11 & $0,10^{*}$ & \\
\hline Medicamentos para la depresión & $-1,62$ & $-2,53$ & $-0,71$ & $-0,17 * * *$ & \\
\hline Medicamentos para el dolor & $-0,01$ & $-1,42$ & 1,39 & $1 \cdot 10^{-2}$ & \\
\hline Medicamentos para la relajación o el sueño & $-0,86$ & $-1,88$ & 0,14 & $-0,08$ & \\
\hline \multicolumn{6}{|l|}{2} \\
\hline Test de prensión manual (kg) & 0,08 & 0,02 & 0,15 & $0,12 *$ & $0,06^{*}$ \\
\hline
\end{tabular}

En el paso 1, se incluyeron las variables confusoras y en el paso 2, la variable independiente de interés.

B y $\beta$, Coeficientes de regresión no estandarizados y estandarizados con los niveles de significación basados en el estadístico t, respectivamente; IC, Intervalo de Confianza; Adj.R2, R2 ajustado con los niveles de significación basados en el cambio de F.

$* \mathrm{p}<0,05 ; * * \mathrm{p}<0,01 ; * * * \mathrm{p}<0,001$ 
Maestre-Cascales, C.; Girela-Rejón, M. J.; Sánchez-Gallo, D. P.; Acosta-Manzano, D.; Gavilán-Carrera, B.; García-Rodríguez, I.; Rojo-González, J. J., y Aparicio, V. A. (2019). Asociación entre fuerza de prensión manual y bienestar en mujeres con fibromialgia. RICYDE. Revista internacional de ciencias del deporte. 58(15), 307-322. https://doi.org/10.5232/ricyde2019.05801

\section{Discusión}

En la fibromialgia, la importancia del bienestar subjetivo, en concreto, el afecto positivo cuya finalidad es alcanzar una mejor salud, ha sido estudiado previamente (Hassett y col., 2008; van Middendorp y col., 2010), insistiendo en la importancia de identificar posibles factores determinantes del bienestar subjetivo. Por lo tanto, el objetivo de este estudio fue analizar la asociación entre la fuerza de prensión manual, considerado como un marcador de salud, con el bienestar subjetivo en mujeres con fibromialgia. Nuestro estudio mostró una asociación entre los mayores niveles de fuerza de prensión manual y puntuaciones más favorables en relación con el bienestar subjetivo (mayor afecto positivo, menor afecto negativo y mayor satisfacción con la vida). Aun siendo significativa, la fuerza de estas asociaciones fue en general débil. Estos hallazgos sugieren que la fuerza podría favorecer al bienestar subjetivo, lo que requiere ser corroborado en estudios longitudinales y experimentales.

La gran mayoría de los estudios previos que han analizado la asociación entre la condición física, en concreto los niveles de fuerza, y los síntomas de la fibromialgia, se han centrado en la sintomatología física (Aparicio y col., 2011; Koklu, Sarigul, Ozisler, Sirzai y Ozel, 2016; Mannerkorpi y col., 2006; Soriano-Maldonado, Henriksen, Segura-Jimenez, Aparicio, Carbonell-Baeza, Delgado-Fernandez y Ruiz, 2015; Tomas-Carus, Gusi, Hakkinen, Hakkinen, Raimundo, y Ortega-Alonso, 2009), demostrando que los niveles de fuerza más altos se asocian con niveles más saludables en relación con el impacto, dolor y funcionamiento físico y vital (Aparicio y col., 2011; Mannerkorpi y col., 2006). Resultados similares se han obtenido para otras variables relacionadas con la calidad de vida como, mayores niveles de fuerza y salud física y mental en estos pacientes (Cordoba-Torrecilla, Aparicio, Soriano-Maldonado, EstevezLopez, Segura-Jimenez, Alvarez-Gallardo, y Delgado-Fernandez, 2016; Soriano-Maldonado, Artero, Segura-Jimenez, Aparicio, Estévez-López, y Alvarez-Gallardo, 2016; SorianoMaldonado, Estévez-López, Segura-Jiménez, Aparicio, Alvarez-Gallardo, HerradorColmenero, y Delgado-Fernández, 2015; Tomas-Carus y col., 2009). También hay evidencias que asocian mayores niveles de condición física con menores de fatiga y rigidez muscular, así como mayor densidad mineral ósea (Estévez-López y col., 2015; Aparicio y col., 2015; CastroPiñero y col., 2017; Soriano-Maldonado, Ruiz y col., 2015). Por lo tanto, nuestros resultados apoyan que alcanzar mayores niveles de fuerza puede tener un efecto beneficioso no solo en la salud física sino también en la salud psicológica en mujeres con fibromialgia.

Centrándonos en síntomas psicológicos, investigaciones previas han observado que niveles más altos de fuerza muscular se asociaron con mejor rendimiento cognitivo en mujeres con fibromialgia (Soriano-Maldonado y col., 2016) y menor depresión en personas diagnosticadas de fibromialgia (Sener, Ucok, Ulasli, Genc, Karabacak, Coban, y Cevik, 2016). Respecto a la ansiedad, un estudio obtuvo asociaciones significativas inversas entre la fuerza muscular y los niveles de ansiedad en las mujeres con fibromialgia (Cordoba-Torrecilla y col., 2016). Por lo tanto, nuestro estudio, extiende el posible papel beneficioso de tener una mejor condición física, en concreto mayores niveles de fuerza desde los aspectos psicológicos negativos (por ejemplo, ansiedad y depresión) a los positivos, como es el bienestar subjetivo.

Aunque este trabajo no se centra en estudiar los mecanismos de las asociaciones analizadas, estudios previos han indicado que la actividad física puede promover una mejor salud mental a través de, al menos, tres mecanismos distintos (neurobiológicos, psicosociales y comportamentales). Así, se ha sugerido que la actividad física puede promover aumentos en los niveles de norepinefrina, serotonina y dopamina en el cerebro, todos ellos asociados con sentimientos de felicidad (Mathew y Paulose, 2011); i.e., mecanismos neurobiológicos. Además, diversos estudios han mostrado que realizar actividad física promueve sentimientos 
Maestre-Cascales, C.; Girela-Rejón, M. J.; Sánchez-Gallo, D. P.; Acosta-Manzano, D.; Gavilán-Carrera, B.; García-Rodríguez, I.; Rojo-González, J. J., y Aparicio, V. A. (2019). Asociación entre fuerza de prensión manual y bienestar en mujeres con fibromialgia. RICYDE. Revista internacional de ciencias del deporte. 58(15), 307-322. https://doi.org/10.5232/ricyde2019.05801

positivos (por ejemplo, Kanning y Schlicht, 2010) posiblemente relacionados con la capacidad de afrontar satisfactoriamente las actividades de la vida diaria que requieren ciertos niveles de condición física; i.e., mecanismos psicosociales. En concreto, la fuerza muscular puede ayudar a satisfacer estas necesidades y conducir así a un mayor bienestar subjetivo en las mujeres con fibromialgia. Por último, participan regularmente en actividad física puede promover la adquisición de otros hábitos de vida saludables (i.e., mecanismos comportamentales) como, por ejemplo, seguir una dieta saludable o una buena higiene de sueño lo cual puede favorecer mayores niveles de bienestar (Borges-Cosic, Aparicio, Estévez-López, Soriano-Maldonado, Acosta-Manzano, Gavilán-Carrera, y Segura-Jiménez, 2019; Carvalho, Ronca, Michels, Huybrechts, Cuenca-Garcia, Marcos, y Carvalho, 2018; Palagini, Carmassi, Conversano, Gesi, Bazzichi, Giacomelli, y Dell'Osso, 2016; Ruiz-Cabello, Soriano-Maldonado, DelgadoFernandez, Alvarez-Gallardo, Segura-Jimenez, Estevez-Lopez, y Aparicio, 2017)

Las recomendaciones actuales para el tratamiento de la fibromialgia indican que el primer paso debe ser realizar ejercicio físico (Clauw, 2014). Así, la European League Against Rheumatism (EULAR) recientemente ha indicado que el ejercicio físico es la única terapia que tiene un grado de evidencia fuerte en esta patología (Macfarlane y col., 2017). Si la causalidad de nuestros resultados es corroborada en futuros estudios, nuestros hallazgos podrían tener implicaciones clínicas. En concreto, nuestros resultados sugieren que aquellos programas de ejercicio físico que mejoren los niveles de fuerza podrían no solo aportar beneficios físicos sino también psicológicos (i.e., mayores niveles de bienestar subjetivo) para las mujeres con fibromialgia.

Este estudio presenta algunas limitaciones. En primer lugar, la naturaleza transversal de nuestro estudio impide la interpretación causal de los resultados. Además, los tamaños del efecto $\left(\mathrm{R}^{2}\right.$ ajustado) de las asociaciones estudiadas fueron aparentemente pequeños. Sin embargo, dado que en el contexto de la salud mental se desconoce cuáles son los puntos de corte de dichos tamaños del efecto para sugerir relevancia clínica, no se puede interpretar la magnitud del tamaño del efecto (Rodríguez-Ayllon, Acosta-Manzano, Coll-Risco, Romero-Gallardo, Borges-Cosic, Estévez-López, y Aparicio, 2019). En segundo lugar, las mujeres con fibromialgia del sur de España podrían estar más afectadas que las residentes en otros países desarrollados como los Países Bajos (Ruiz-Montero, Van Wilgen, Segura-Jiménez, CarbonellBaeza, y Delgado-Fernández, 2015) o Estados Unidos (Toussaint, Vincent, McAllister, Oh, y Hassett, 2014). En tercer lugar, debido al pequeño tamaño muestral de los hombres, en este estudio solo se incluyeron mujeres. Por lo tanto, nuestros resultados no pueden ser extrapolables a otras poblaciones ni a hombres con fibromialgia. Para confirmar el hallazgo principal del presente estudio, sería interesante replicarlo en estudios futuros con muestras independientes. En cambio, como principal fortaleza del presente estudio, destacamos el tamaño muestral con la que se ha trabajado, siendo representativa de la población Andaluza de mujeres con fibromialgia (Segura-Jimenez y col., 2015a). Además, el equipo de investigación corroboró el diagnóstico de fibromialgia de los participantes.

\section{Conclusiones}

Nuestros hallazgos sugieren que las mujeres con fibromialgia con mayores niveles de fuerza indicaron tener mayores niveles de bienestar subjetivo, en concreto mayores de afecto positivo y satisfacción con la vida, así como menores de afecto negativo. En general, la fuerza de estas asociaciones fue débil. Si futuros estudios experimentales confirman la causalidad de nuestros hallazgos, los programas de ejercicio que mejoren los niveles de fuerza podrían suponer de forma efectiva el aumento del bienestar subjetivo en las mujeres con fibromialgia. En el caso 
Maestre-Cascales, C.; Girela-Rejón, M. J.; Sánchez-Gallo, D. P.; Acosta-Manzano, D.; Gavilán-Carrera, B.; García-Rodríguez, I.; Rojo-González, J. J., y Aparicio, V. A. (2019). Asociación entre fuerza de prensión manual y bienestar en mujeres con fibromialgia. RICYDE. Revista internacional de ciencias del deporte. 58(15), $307-322$. https://doi.org/10.5232/ricyde2019.05801

de producirse dichos aumentos, los futuros estudios deben evaluar la relevancia clínica de sus hallazgos.

\section{Referencias}

Aparicio, V. A.; Carbonell-Baeza, A.; Ruiz, J. R.; Aranda, P.; Tercedor, P.; DelgadoFernandez, M., \& Ortega, F. B. (2013). Fitness testing as a discriminative tool for the diagnosis and monitoring of fibromyalgia. Scandinavian Journal of Medicine \& Science in Sports, 23(4), 415-423.

https://doi.org/10.1111/j.1600-0838.2011.01401.x

Aparicio, V. A.; Ortega, F. B.; Heredia, J. M.; Carbonell-Baeza, A.; Sjöström, M., \& Delgado-Fernandez, M. (2011). Handgrip strength test as a complementary tool in the assessment of fibromyalgia severity in women. Archives of Physical Medicine and Rehabilitation, 92(1), 83-88.

https://doi.org/10.1016/j.apmr.2010.09.010

Aparicio, V. A.; Segura-Jimenez, V.; Alvarez-Gallardo, I. C.; Soriano-Maldonado, A.; Castro-Pinero, J.; Delgado-Fernandez, M., \& Carbonell-Baeza, A. (2015). Fitness testing in the fibromyalgia diagnosis: the al-Ándalus project. Medicine and Science in Sports and Exercise, 47(3), 451-459.

Borges-Cosic, M., Aparicio, V. A., Estévez-López, F., Soriano-Maldonado, A., AcostaManzano, P., Gavilán-Carrera, B., ... Segura-Jiménez, V. (2019). Sedentary time, physical activity, and sleep quality in fibromyalgia: The al-Ándalus project. Scandinavian Journal of Medicine \& Science in Sports, 29(2), 266-274.

https://doi.org/10.1111/sms.13318

Carvalho, K., Ronca, D., Michels, N., Huybrechts, I., Cuenca-Garcia, M., Marcos, A., ... Carvalho, L. (2018). Does the Mediterranean Diet Protect against Stress-Induced Inflammatory Activation in European Adolescents? The HELENA Study. Nutrients, 10(11), 1770.

https://doi.org/10.3390/nu10111770

Castro-Piñero, J.; Aparicio, V. A.; Estévez-López, F.; Álvarez-Gallardo, I. C.; Borges-Cosic, M.; Soriano-Maldonado, A. ... Segura-Jiménez, V. (2017). The Potential of Established Fitness Cut-off Points for Monitoring Women with Fibromyalgia: The al-Ándalus Project. International Journal of Sports Medicine, 38(05), 359-369.

Clauw, D. J. (2014). Fibromyalgia: a clinical review. Jama, 311(15), 1547-1555.

Cordoba-Torrecilla, S.; Aparicio, V. A.; Soriano-Maldonado, A.; Estevez-Lopez, F.; SeguraJimenez, V.; Alvarez-Gallardo, I., ... Delgado-Fernandez, M. (2016). Physical fitness is associated with anxiety levels in women with fibromyalgia: the al-Andalus project. Quality of Life Research, 25(4), 1053-1058.

https://doi.org/10.1007/s11136-015-1128-y

Diener, E., Suh, E. M.; Lucas, R. E., \& Smith, H. L. (1999). Subjective well-being: Three decades of progress. Psychological Bulletin, 125(2), 276.

Estévez-López, F.; Gray, C. M.; Segura-Jiménez, V.; Soriano-Maldonado, A.; ÁlvarezGallardo, I. C.; Arrayás-Grajera, M. J., ... Pulido-Martos, M. (2015). Independent and combined association of overall physical fitness and subjective well-being with fibromyalgia severity: the al-Ándalus project. Quality of Life Research, 24(8), 18651873.

Estevez-Lopez, F.; Segura-Jimenez, V.; Alvarez-Gallardo, I. C.; Borges-Cosic, M.; PulidoMartos, M.; Carbonell-Baeza, A.; ... Delgado-Fernandez, M. (2017). Adaptation profiles comprising objective and subjective measures in fibromyalgia: the al-Andalus project. Rheumatology, 56(11), 2015-2024.

https://doi.org/10.1093/rheumatology/kex302. 
Maestre-Cascales, C.; Girela-Rejón, M. J.; Sánchez-Gallo, D. P.; Acosta-Manzano, D.; Gavilán-Carrera, B.; García-Rodríguez, I.; Rojo-González, J. J., y Aparicio, V. A. (2019). Asociación entre fuerza de prensión manual y bienestar en mujeres con fibromialgia. RICYDE. Revista internacional de ciencias del deporte. 58(15), 307-322. https://doi.org/10.5232/ricyde2019.05801

Estevez-Lopez, F. (2018). Fibromyalgia: adapted, positive, and fit people: Breaking stereotypes! (Tesis doctoral). Utrecht University, Utrecht, Países Bajos.

Hassett, A. L.; Simonelli, L. E.; Radvanski, D. C.; Buyske, S.; Savage, S. V, \& Sigal, L. H. (2008). The relationship between affect balance style and clinical outcomes in fibromyalgia. Arthritis Care \& Research: Official Journal of the American College of Rheumatology, 59(6), 833-840.

Kanning, M., \& Schlicht, W. (2010). Be active and become happy: an ecological momentary assessment of physical activity and mood. Journal of Sport and Exercise Psychology, 32(2), 253-261.

Koklu, K.; Sarigul, M.; Ozisler, Z.; Sirzai, H., \& Ozel, S. (2016). Handgrip Strength in Fibromyalgia. Archives of Rheumatology, 31(2), 158-161. https://doi.org/10.5606/ArchRheumatol.2016.5736

Koltyn, K. F. (1997). The thermogenic hypothesis. In W. P. Morgan (Ed.), Series in health psychology and behavioral medicine. Physical activity and mental health (pp. 213-226). Philadelphia, PA, US: Taylor \& Francis.

Lobo, A.; Ezquerra, J.; Gómez, F. B.; Sala, J. M., \& Seva, A. D. (1979). Cognocitive minitest (a simple practical test to detect intellectual changes in medical patients). Actas Luso-Espanolas de Neurologia, Psiquiatria y Ciencias Afines, 7(3), 189-202.

López, F. E. (2018). Gene-physical activity interaction with pain, fatigue, and resilience in women with fibromyalgia (Tesis doctoral). Universidad de Granada, Granada, España.

Macfarlane, G. J.; Kronisch, C.; Dean, L. E.; Atzeni, F.; Haeuser, W.; Fluss, E., ... Jones, G. T. (2017). EULAR revised recommendations for the management of fibromyalgia. Annals of the Rheumatic Diseases, 76(2), 318-328. https://doi.org/10.1136/annrheumdis-2016-209724

Mannerkorpi, K.; Svantesson, U., \& Broberg, C. (2006). Relationships between performance-based tests and patients' ratings of activity limitations, self-efficacy, and pain in fibromyalgia. Archives of Physical Medicine and Rehabilitation, 87(2), 259-264. https://doi.org/10.1016/j.apmr.2005.10.013

Mathew, J., \& Paulose, C. S. (2011). The healing power of well-being. Acta Neuropsychiatrica, 23(4), 145-155.

Nordenskiold, U. M., \& Grimby, G. (1993). Grip force in patients with rheumatoid-arthritis and fibromyalgia and in healthy-subjects - a study with the grippit instrument. Scandinavian Journal of Rheumatology, 22(1), 14-19.

Palagini, L., Carmassi, C., Conversano, C., Gesi, C., Bazzichi, L., Giacomelli, C., \& Dell'Osso, L. (2016). Transdiagnostic factors across fibromyalgia and mental disorders: sleep disturbances may play a key role. A clinical review. Clinical and Experimental Rheumatology, 34(2 Suppl 96), S140-4. Retrieved from: http://www.ncbi.nlm.nih.gov/pubmed/27157399

Rodriguez-Ayllon, M., Acosta-Manzano, P., Coll-Risco, I., Romero-Gallardo, L., BorgesCosic, M., Estévez-López, F., \& Aparicio, V. (2019). Journal of Sport and Health Science (en prensa).

Ruiz-Montero, P. J.; Van Wilgen, C. P.; Segura-Jiménez, V.; Carbonell-Baeza, A., \& Delgado-Fernández, M. (2015). Illness perception and fibromyalgia impact on female patients from Spain and the Netherlands: do cultural differences exist? Rheumatology International, 35(12), 1985-1993.

Ruiz, J. R.; España-Romero, V.; Ortega, F. B.; Sjöström, M.; Castillo, M. J., \& Gutierrez, A. (2006). Hand span influences optimal grip span in male and female teenagers. The Journal of Hand Surgery, 31(8), 1367-1372. 
Maestre-Cascales, C.; Girela-Rejón, M. J.; Sánchez-Gallo, D. P.; Acosta-Manzano, D.; Gavilán-Carrera, B.; García-Rodríguez, I.; Rojo-González, J. J., y Aparicio, V. A. (2019). Asociación entre fuerza de prensión manual y bienestar en mujeres con fibromialgia. RICYDE. Revista internacional de ciencias del deporte. 58(15), $307-322$. https://doi.org/10.5232/ricyde2019.05801

Ruiz-Cabello, P., Soriano-Maldonado, A., Delgado-Fernandez, M., Alvarez-Gallardo, I. C., Segura-Jimenez, V., Estevez-Lopez, F., ... Aparicio, V. A. (2017). Association of Dietary Habits with Psychosocial Outcomes in Women with Fibromyalgia: The al-Ándalus Project. Journal of the Academy of Nutrition and Dietetics, 117(3), 422-432.e1. https://doi.org/10.1016/j.jand.2016.09.023

Segura-Jimenez, V.; Alvarez-Gallardo, I. C.; Carbonell-Baeza, A.; Aparicio, V. A.; Ortega, F. B.; Casimiro, A. J., \& Delgado-Fernandez, M. (2015a). Fibromyalgia has a larger impact on physical health than on psychological health, yet both are markedly affected: The al-Andalus project. Seminars in Arthritis and Rheumatism, 44(5), 563-570. https://doi.org/10.1016/j.semarthrit.2014.09.010

Sener, U.; Ucok, K.; Ulasli, A. M.; Genc, A.; Karabacak, H.; Coban, N. F., ... Cevik, H. (2016). Evaluation of health-related physical fitness parameters and association analysis with depression, anxiety, and quality of life in patients with fibromyalgia. International Journal of Rheumatic Diseases, 19(8), 763-772. https://doi.org/10.1111/1756-185x.12237

Sicras-Mainar, A.; Rejas, J.; Navarro, R.; Blanca, M.; Morcillo, Á.; Larios, R., ... Villarroya, C. (2009). Treating patients with fibromyalgia in primary care settings under routine medical practice: a claim database cost and burden of illness study. Arthritis Research \& Therapy, 11(2), R54.

Soriano-Maldonado, A.; Artero, E. G.; Segura-Jimenez, V.; Aparicio, V. A.; Estevez-Lopez, F.; Alvarez-Gallardo, I. C., ... al-Andalus Project Res, G. (2016). Association of physical fitness and fatness with cognitive function in women with fibromyalgia. Journal of Sports Sciences, 34(18), 1731-1739.

https://doi.org/10.1080/02640414.2015.1136069

Soriano-Maldonado, A.; Estévez-López, F.; Segura-Jiménez, V.; Aparicio, V. A.; AlvarezGallardo, I. C.; Herrador-Colmenero, M. ; ... Delgado-Fernández, M. (2015). Association of physical fitness with depression in women with fibromyalgia. Pain Medicine, 17(8), 1542-1552.

Soriano-Maldonado, A., Henriksen, M.; Segura-Jimenez, V.; Aparicio, V. A.; CarbonellBaeza, A.; Delgado-Fernandez, M., ... Ruiz, J. R. (2015). Association of Physical Fitness With Fibromyalgia Severity in Women: The al-Andalus Project. Archives of Physical Medicine and Rehabilitation, 96(9), 1599-1605.

https://doi.org/10.1016/j.apmr.2015.03.015

Soriano-Maldonado, A.; Ruiz, J. R.; Aparicio, V. A.; Estevez-Lopez, F.; Segura-Jimenez, V.; Alvarez-Gallardo, I. C., ... Ortega, F. B. (2015). Association of Physical Fitness With Pain in Women With Fibromyalgia: The al-andalus Project. Arthritis Care \& Research, 67(11), 1561-1570.

https://doi.org/10.1002/acr.22610

Stanton, A. L.; Revenson, T. A., \& Tennen, H. (2007). Health psychology: psychological adjustment to chronic disease. Annu. Rev. Psychol., 58, 565-592.

Tomas-Carus, P.; Gusi, N.; Hakkinen, A.; Hakkinen, K.; Raimundo, A., \& Ortega-Alonso, A. (2009). Improvements of muscle strength predicted benefits in HRQOL and postural balance in women with fibromyalgia: an 8-month randomized controlled trial. Rheumatology, 48(9), 1147-1151.

https://doi.org/10.1093/rheumatology/kep208

Toussaint, L. L.; Vincent, A.; McAllister, S. J.; Oh, T. H., \& Hassett, A. L. (2014). A comparison of fibromyalgia symptoms in patients with healthy versus depressive, low and reactive affect balance styles. Scandinavian Journal of Pain, 5(3), 161-166. 
Valkeinen, H.; Alen, M.; Hakkinen, A.; Hannonen, P.; Kukkonen-Harjula, K., \& Hakkinen, K. (2008). Effects of concurrent strength and endurance training on physical fitness and symptoms in postmenopausal women with fibromyalgia: A randomized controlled trial. Archives of Physical Medicine and Rehabilitation, 89(9), 1660-1666. https://doi.org/10.1016/j.apmr.2008.01.022

van Middendorp, H.; Lumley, M. A.; Jacobs, J. W. G.; Bijlsma, J. W. J., \& Geenen, R. (2010). The effects of anger and sadness on clinical pain reports and experimentallyinduced pain thresholds in women with and without fibromyalgia. Arthritis Care \& Research, 62(10), 1370-1376.

van Middendorp, H.; Lumley, M. A.; Jacobs, J. W. G.; van Doornen, L. J. P.; Bijlsma, J. W. J., \& Geenen, R. (2008). Emotions and emotional approach and avoidance strategies in fibromyalgia. Journal of Psychosomatic Research, 64(2), 159-167.

Watson, D.; Clark, L. A., \& Tellegen, A. (1988). Development and validation of brief measures of positive and negative affect: the PANAS scales. Journal of Personality and Social Psychology, 54(6), 1063.

Wolfe, F.; Smythe, H. A.; Yunus, M. B.; Bennett, R. M.; Bombardier, C.; Goldenberg, D. L., ... Clark, P. (1990). The American College of Rheumatology 1990 criteria for the classification of fibromyalgia. Arthritis \& Rheumatism: Official Journal of the American College of Rheumatology, 33(2), 160-172. 